\title{
Additions to Pseudocamarosporium; two new species from Italy
}

\author{
Wijayawardene $\mathrm{NN}^{1,2}$, Tibpromma $\mathrm{S}^{2}$, Hyde $\mathrm{KD}^{2,3,4}$, An $\mathrm{YL}^{5}$, Camporesi $\mathrm{E}^{6}$ and \\ Wang $\mathbf{Y}^{1,2 *}$
}

\begin{abstract}
${ }^{1}$ Department of Plant Pathology, Agriculture College, Guizhou University, 550025,
${ }^{2}$ Center of Excellence in Fungal Research, and School of Science, Mae Fah Luang University, Chiang Rai, 57100 , Thailand

${ }^{3}$ Key Laboratory for Plant Diversity and Biogeography of East Asia, Kunming Institute of Botany, Chinese Academy of Sciences, 132 Lanhei Road, Kunming 650201, P.R. China

${ }^{4}$ World Agroforestry Centre, East Asia Office, Kunming 650201, Yunnan, P.R. China

${ }^{5}$ P.R. China The Key Laboratory of Karst Environment and Geohazard Prevention, Ministry of Education, Guizhou University

${ }^{6}$ A.M.B. Gruppo Micologico Forlivese “Antonio Cicognani”, Via Roma 18, Forlì, Italy; A.M.B. Circolo Micologico “Giovanni Carini”, C.P. 314, Brescia, Italy; Società per gli Studi Naturalistici della Romagna, C.P. 144, Bagnacavallo (RA), Italy
\end{abstract}

Wijayawardene NN, Tibpromma S, Hyde KD, An YL, Camporesi E, Wang Y 2016 - Additions to Pseudocamarosporium; two new species from Italy. Studies in Fungi 1(1), 1-10, Doi $10.5943 / \mathrm{sif} / 1 / 1 / 1$

\begin{abstract}
Two coelomycetous taxa with muriform conidia were collected from Italy, and subjected to morpho-molecular taxonomic analyses. A mega blast search showed that the new taxa had a close relationship with Pseudocamarosporium. Maximum-likelihood and Bayesian analyses of combined LSU, SSU and ITS sequence data also showed that these strains reside in Didymosphaeriaceae and cluster with Pseudocamarosporium sensu stricto. Following detailed morphological and molecular analyses, these are introduced as new species in Pseudocamarosporium. The new taxa are illustrated and compared with other known species in the genus.
\end{abstract}

Key words - Camarosporium - Coelomycetous fungi - Muriform - Phylogeny

\section{Introduction}

Phylogenetic studies based on analyses of DNA sequence data showed that camarosporiumlike taxa are polyphyletic in Pleosporales and therefore Wijayawardene et al. (2014c) introduced two new genera, viz. Paracamarosporium and Pseudocamarosporium, to accommodate camarosporium-like taxa in Didymosphaeriaceae. Both Paracamarosporium and Pseudocamarosporium have pycnidial conidiomata, enteroblastic and phialidic conidiogenesis with percurrent proliferation and muriform conidia (Wijayawardene et al. 2014c). However, Paracamarosporium is distinct in having hyaline, smooth-walled, guttulate, bacilliform to subcylindrical microconidia (Crous et al. 2013 as Camarosporium psoraleae Crous \& M.J. Wingf.). Crous et al. (2015) showed that Paraconiothyrium africanum Damm et al. grouped in Pseudocamarosporium sensu stricto, thus, they treated it as a species of Pseudocamarosporium.

We collected two coelomycetous taxa with muriform conidia from Italy, on Quercus pubescens and Pinus nigra. These taxa are morphologically, similar to Camarosporium sensu stricto, but a megablast search using LSU rDNA sequence data, showed that they belong in Submitted 2 December 2015, Accepted 28 January 2016, Published online 17 February 2016 
Didymosphaeriaceae, Massarineae. Maximum-likelihood and Bayesian analyses of combined LSU, SSU and ITS gene regions show these strains grouping with Pseudocamarosporium sensu stricto. Hence, two new species are introduced based on morphological characters and phylogenetic analyses.

\section{Materials \& methods}

\section{Collection, isolation and morphological studies}

Decayed plant material collected in Italy, were placed in paper bags and/or Zip-lock bags, and brought to the laboratory. The samples were observed first under a stereoscope to locate fungal taxa. Squash mounts were made to reveal the morphology of conidiophores, type of conidiogenous cells and conidiogenesis and morphology of conidia (Sutton 1980). Thin hand-sections of conidioma were made by razor blade to examine the shape of conidiomata and arrangement of conidiophores and conidiogenous cells. Morphological characters were examined under a compound microscope (Nikon Eclipse E600 DIC microscope and a Nikon DS-U2 camera or a Nikon Eclipse 80i compound microscope fitted with a Canon 450D digital camera).

Single conidial isolation used the method in Chomnunti et al. (2014) and germinating conidia were transferred aseptically to potato dextrose agar (PDA). Germinating conidia were transferred to PDA plates and incubated at $18{ }^{\circ} \mathrm{C}$ for further growth. Colony colour and other characters were assessed after 1 to 2 weeks. The holotypes are deposited in the Mae Fah Luang University Herbarium (MFLU), Chiang Rai, Thailand, Herbarium of the Department of Plant Pathology, Agricultural College, Guizhou University (HGUP) and Kunming Institute of Botany, Chinese Academy of Sciences, Kunming, P.R. China (HKAS). Ex-type cultures are also deposited in Culture Collection at Mae Fah Luang University (MFLUCC) and Department of Plant Pathology, Agriculture College, Guizhou University, China (GUCC). Facesoffungi (Jayasiri et al. 2015) and Index Fungorum (2016) numbers were provided for new taxa.

\section{DNA extraction, PCR amplification and sequencing}

Colonies generated from germinated single conidia were further grown on PDA for 14 days at $18{ }^{\circ} \mathrm{C}$. Fresh fungal mycelia were scraped from PDA using sterilized scalpels. A BIOMIGA Fungus Genomic DNA Extraction Kit (GD2416) was used to extract DNA from the scraped mycelia. The amplification of rDNA regions of the internal transcribed spacers (ITS), small subunit rDNA (SSU) and large subunit (LSU) genes was carried out by using primers ITS5 and ITS4, NS1 and NS4 and LROR and LR5 (Vilgalys \& Hester 1990, White et al. 1990). Optimum conditions for amplification of ITS and LSU regions are as described in Alves et al. $(2004,2005)$ and for the SSU region as demonstrated in Phillips et al. (2008). Amplified PCR fragments were checked on 1\% agarose electrophoresis gels stained with ethidium bromide. Purified PCR products (by minicolumns, purification resin and buffer according to the manufacturer's protocols Amersham product code: 27-9602-01) were sent to SinoGenoMax Co., Beijing, China for DNA sequencing. The nucleotide sequence data obtained are submitted to GenBank (Table 1).

\section{Phylogenetic analyses}

A megablast search was carried out to confirm the placement of the new strains in Pleosporales and therefore phylogenetically related sequences were downloaded from GenBank (Table 1). Since, both strains showed a closer relationship with Pseudocamarosporium, molecular data analyses in Hyde et al. (2013), Ariyawansa et al. (2014) and Wijayawardene et al. (2014c, 2016) were used to select c strains in Didymosphaeriaceae. Sequences for each gene region (LSU, SSU and ITS) were aligned using MAFFTv6 (Katoh et al. 2002, Katoh \& Toh 2008), and online sequence alignment was edited under the default settings (mafft.cbrc.jp/alignment/server/). All absent genes were coded as missing data.

Both datasets were performed using maximum likelihood (ML) and Bayesian Posterior Probabilities (BYPP). Maximum-likelihood (ML) analyses was performed in RAxML (Stamatakis 
2006) implemented in raxmlGUI v.0.9b2 (Silvestro \& Michalak 2010). The model of evolution was estimated by using MrModeltest 2.2 (Nylander 2004). Independent Bayesian phylogenetic analyses were performed in MrBayes v. 3.1.2 (Huelsenbeck \& Ronquist 2001) using a uniform [GTR $+\mathrm{I}+\mathrm{G}]$ model, lset $n s t=6$ rates $=$ invgamma; prsetstatefreqpr $=$ dirichlet $(1,1,1,1)$. Posterior probabilities (PP) (Rannala \& Yang 1996, Zhaxybayeva \& Gogarten 2002) were determined by Markov Chain Monte Carlo sampling (BMCMC) in MrBayes v. 3.0b4 (Huelsenbeck \& Ronquist 2001). Six simultaneous Markov chains were run for 10,000,000 generations and trees were sampled every 100th generation (resulting in 10,000 total trees). Phylogenetic trees were visualized with FigTree (Rambaut 2012). BS values of ML (equal or above 70\%) and BYPP with those equal or greater than 0.95 of each node are shown on the upper branches.

Table 1 Strains used in this study. Type strains are in bold and newly generated sequences are in bold and marked with an asterisk

\begin{tabular}{|c|c|c|c|c|}
\hline \multirow{2}{*}{ Taxon } & \multirow{2}{*}{$\begin{array}{l}\text { Culture } \\
\text { collection } \\
\text { number }\end{array}$} & \multicolumn{2}{|c|}{ GenBank Accession number } & \multirow[b]{2}{*}{ ITS } \\
\hline & & LSU & SSU & \\
\hline Alloconiothyrium aptrooti & CBS 981.95 & JX496235 & & \\
\hline Alloconiothyrium aptrootii & CBS 980.95 & JX496234 & & JX496121 \\
\hline Austropleospora archidendri & CBS 168.77 & JX496162 & & JX496049 \\
\hline Austropleospora osteospermi & LM-2009a & & & FJ481946 \\
\hline Deniquelata barringtoniae & MFLUCC 11-0422 & JX254655 & JX254656 & JX254654 \\
\hline Deniquelata barringtoniae & MFLUCC 11-0257 & KM214000 & KM214003 & KM213997 \\
\hline Didymosphaeria rubi-ulmifolii & MFLUCC 14-0023 & KJ436586 & KJ436588 & \\
\hline Didymosphaeria rubi-ulmifolii & MFLUCC 14-0024 & & KJ436587 & \\
\hline Kalmusia ebuli & CBS 123120 & JN644073 & JN851818 & KF796674 \\
\hline Kalmusia italica & MFLUCC 13-0066 & KP325441 & KP325442 & KP325440 \\
\hline Massarina eburnea & CBS 13969 & GU301840 & GU296170 & \\
\hline Neokalmusia brevispora & CBS 120248 & JX681110 & & \\
\hline Neokalmusia scabrispora & MAFF 239517 & AB524593 & AB524452 & LC014575 \\
\hline Paracamarosporium fagi & CPC 24892 & KR611905 & & KR611887 \\
\hline Paracamarosporium fagi & CPC 24890 & KR611904 & & KR611886 \\
\hline Paracamarosporium fungicola & CBS 113269 & JX496133 & & JX496020 \\
\hline Paracamarosporium psoraleae & CPC 21632 & KF777199 & KF777143 & \\
\hline Paraconiothyrium brasiliense & CBS 254.88 & JX496171 & & JX496058 \\
\hline $\begin{array}{l}\text { Paraconiothyrium } \\
\text { cyclothyrioides }\end{array}$ & CBS 432.75 & JX496201 & & JX496088 \\
\hline Paraconiothyrium estuarinum & CBS 109850 & JX496129 & AY642522 & JX496016 \\
\hline Paraconiothyrium nelloi & MFLUCC 13-0487 & KP711365 & KP711370 & KP711360 \\
\hline Paraphaeosphaeria angularis & CBS 167.70 & JX496160 & & JX496047 \\
\hline Paraphaeosphaeria michotii & MFLUCC 13-0349 & KJ939282 & KJ939285 & KJ939279 \\
\hline Pseudocamarosporium corni & MFLUCC 13-0541 & KJ813279 & KJ819946 & KJ747048 \\
\hline Pseudocamarosporium lonicerae & MFLUCC 13-0532 & KJ813278 & KJ819947 & KJ747047 \\
\hline Pseudocamarosporium pinicola* & MFLUCC 14-0457 & KT211629 & KT211626 & \\
\hline $\begin{array}{l}\text { Pseudocamarosporium } \\
\text { propinquum }\end{array}$ & MFLUCC 13-0544 & KJ813280 & KJ819949 & KJ747049 \\
\hline $\begin{array}{l}\text { Pseudocamarosporium } \\
\text { quercinum }\end{array}$ & MFLUCC 14-0456 & KT211628 & KT211625 & \\
\hline Pseudocamarosporium tilicola & MFLUCC 13-0550 & KJ813281 & KJ819950 & KJ747050 \\
\hline Verrucoconiothyrium nitidae & CBS 119209 & & & EU552112 \\
\hline
\end{tabular}




\section{Results}

Phylogenetic analyses

A combined dataset of LSU, SSU and ITS gene regions is used for taxa phylogenetically close to Pseudocamarosporium (Wijayawardene et al. 2016). Wijayawardene et al. (2014c) introduced Pseudocamarosporium with five species viz. P. corni Wijayaw. et al., P. lonicerae Wijayaw. et al., P. piceae Wijayaw. et al., P. propinquum (Sacc.) Wijayaw. et al. and P. tiliicola Wijayaw. et al. (Wijayawardene et al. 2014c). Liu et al. (2015) introduced $P$. cotinae Norphanphoun et al. and Crous et al. (2015) introduced $P$. africanum (Damm et al.) Crous and $P$. brabeji (Marincowitz et al.) Crous. Hence, the genus comprises eight species.

The combined LSU, SSU and ITS data set consists of 29 strains with Massarina eburnea (CBS 13969) as outgroup taxon. Pseudocamarosporium sensu stricto grouped as a distinct clade in Didymosphaeriaceae with high bootstrap value in ML analysis and high PP value in Bayesian analyses (98\% and 1.00 respectively) (Fig. 1).

The taxon from Quercus sp. closely grouped with Pseudocamarosporium tilicola with high bootstrap and PP values (85\% and 1.00 respectively). The taxon from Pinus sp. grouped with $P$. barbeji and $P$. corni with low bootstrap and PP values. Pseudocamarosporium africanum and $P$. brabeji do not have SSU sequence data, thus, we could not include them in the analyses. The short branch lengths could resulted from lack of SSU sequence data. Moreover, Crous et al. (2015) used only LSU sequence data which also resulted in poor species distinction in Bayesian analysis.

Paraconiothyrium fungicola resides in Paracamarosporium sensu stricto (Wijayawardene et al. 2014c, Crous et al. 2015) and thus, we transfer it to the genus Paracamarosporium as a new combination.

\section{Taxonomy}

Paracamarosporium fungicola (Verkley \& Wicklow) Wijayaw. \& K.D. Hyde, comb. nov. Index fungorum number: IF551932

Basionym: Paraconiothyrium fungicola Verkley \& Wicklow, in Verkley, da Silva, Wicklow \& Crous, Stud. Mycol. 50(2): 331 (2004)

Pseudocamarosporium quercinum Wijayaw., Camporesi \& K.D. Hyde, sp. nov. Index fungorum number: IF551300

Facesoffungi Number: FoF 00877

Etymology - Named after the generic name of host

Saprobic on dead branches and stems of Quercus pubescens. Sexual morph: Undetermined. Asexual morph: Conidiomata 420-450 $\mu \mathrm{m}$ diam., 250-280 $\mu \mathrm{m}$ high, pycnidial, immersed, erumpent, solitary, globose, unilocular, black, with a long neck. Pycnidial wall 50-70 $\mu \mathrm{m}$ wide, multi-layered, with 3-5 outer layers of brown-walled cells of textura angularis, with inner layer thin, hyaline. Conidiophores reduced to conidiogenous cells. Conidiogenous cells $2-7 \times 3-5 \mu \mathrm{m}$, blastic, phialidic, discrete, determinate, hyaline, smooth. Conidia 15-18 $\times$ 6-7.5 $\mu \mathrm{m}$ (mean $=16.41$ $\times 6.79 \mu \mathrm{m}, \mathrm{n}=20$ ), oblong, mostly straight, occasionally slightly curved, muriform, with $1-4$ transverse and 1-2 longitudinal septa, continuous, initially hyaline, later becoming brown to dark brown at maturity, narrowly rounded at both ends, smooth-walled.

Culture characteristics - Colonies on PDA slow growing, reaching $1.5 \mathrm{~cm}$ diam. after 1 week at $18{ }^{\circ} \mathrm{C}$, dense mycelium, circular, rough margin white at first, greenish white after 1 week, flat or effuse on the surface. Hyphae septate branched, hyaline, thin.

Material examined - Italy, Province of Forlì-Cesena [FC], Pian di Spino - Civitella di Romagna, on dead branches of Quercus pubescens (Fagaceae), 2 December 2013, Erio Camporesi, IT 1552 (MFLU 15-0733, holotype), (HKAS 88741, isotype); living cultures MFLUCC 14-0456 $=$ GUCC 54 . 
Notes - There have been several camarosporium-like taxa reported on Quercus spp. viz. Camarosporium betulinum Died. (14-20 × 5-7.5 $\mu \mathrm{m})$, C. juglandis Ellis \& Barthol. (12-25 $\times 8-12$ $\mu \mathrm{m})$, C. kursanovii Mekht. (7-16.3 × 7-9 $\mu \mathrm{m})$, C. quercus Sacc. \& Roum. $(25-28 \times 8-10 \mu \mathrm{m}), C$. variabile (Berk. \& M.A. Curtis) Sacc. (30-60 $\mu \mathrm{m}$ long) (Ellis \& Ellis 1985, Farr \& Rossman 2016). Our collection is morphologically distinct from all above taxa and in molecular analyses resides in Didymosphaeriaceae with other Pseudocamarosporium species (Fig. 1)

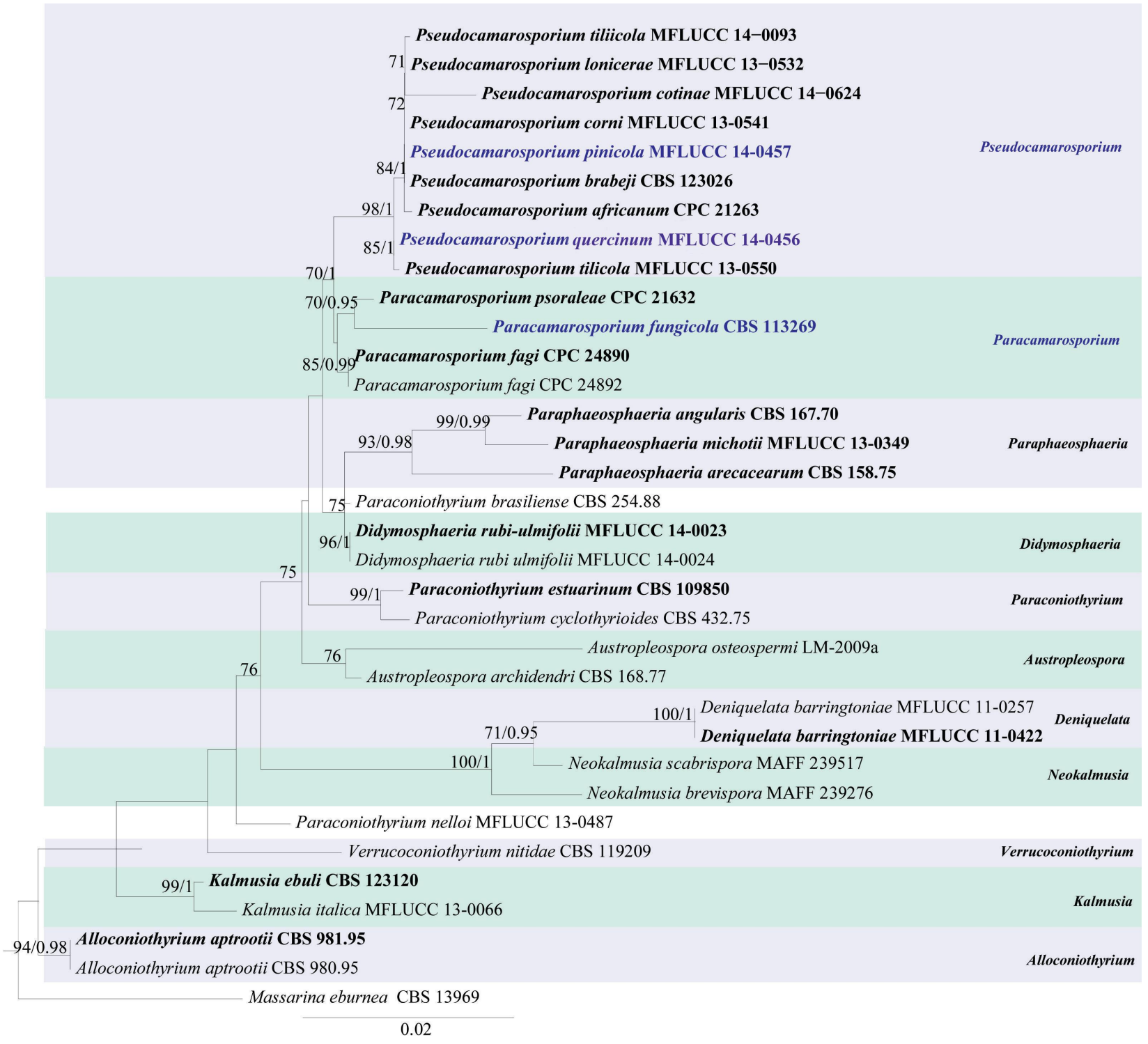

Fig. 1 - The best scoring RAxML tree resulting from the combined analyses of LSU, SSU and ITS sequence data. Bootstrap support values $(>70 \%)$ for maximum-likelihood (ML) and Bayesian posterior probabilities (BYPP) (above 0.95) are given above the nodes. Ex-type strains are in bold and newly introduced species and combinations are in blue.

Pseudocamarosporium pinicola Wijayaw., Camporesi \& K.D. Hyde, sp. nov.

Index fungorum number: IF551302

Facesoffungi Number: FoF 00879

Etymology - Named after the generic name of host

Endophytic or saprobic on dead branch of Pinus nigra. Sexual morph: Undetermined. Asexual morph: Conidiomata 1000-1300 $\mu \mathrm{m}$ diam., 800-1100 $\mu \mathrm{m}$ high, pycnidial, immersed, globose to subglobose, unilocular, solitary, black, with a papillate, centrally located ostiole. Pycnidial wall multi-layered, with a thick outer layer, composed of dark brown cells of textura 


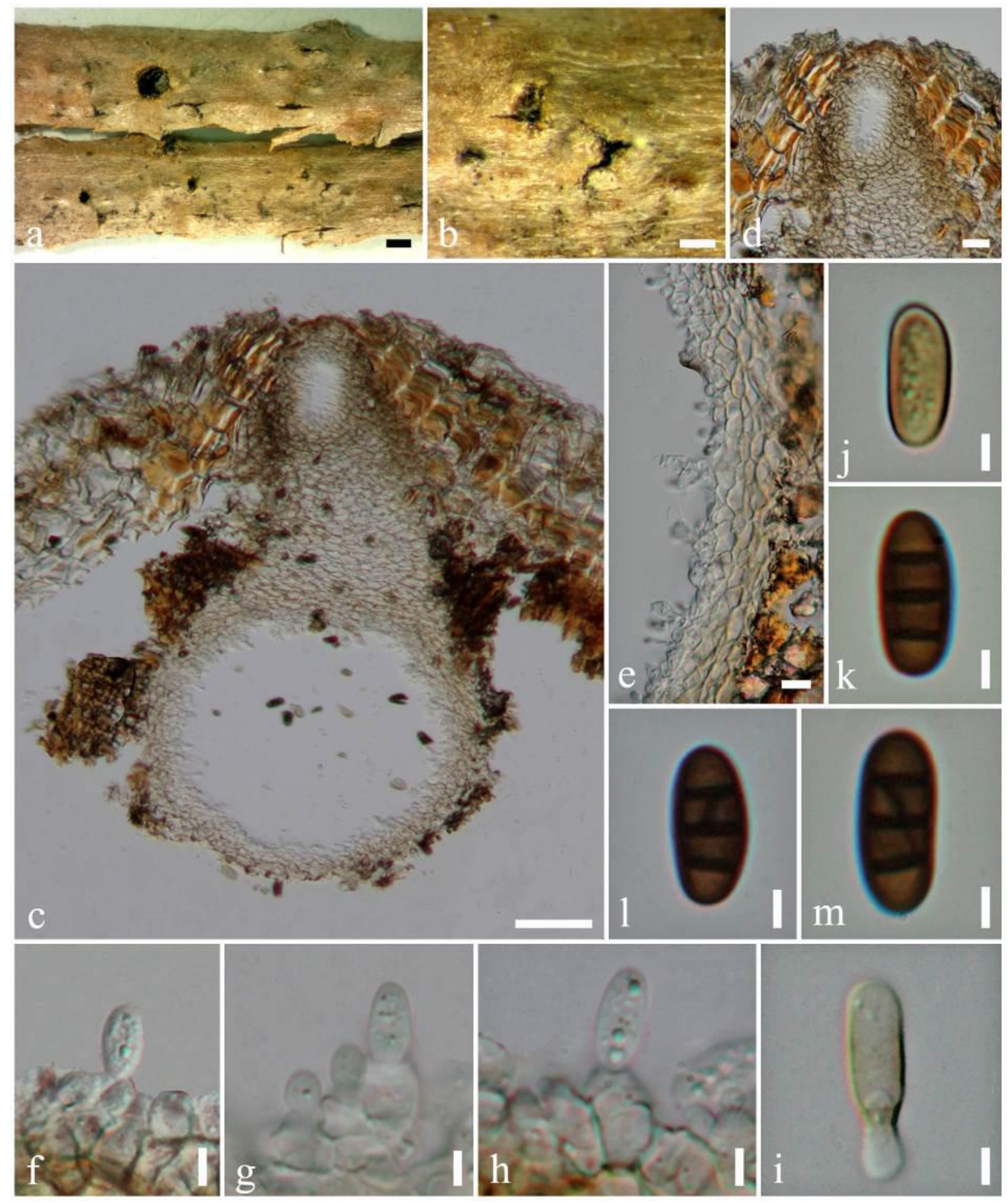

Fig. 2 - Pseudocamarosporium quercinum (holotype) a, b. Immersed conidiomata on dead branches of Quercus pubescens. c. Vertical section of pycnidium. d. Vertical section of the neck of pycnidium. e. Conidioma wall. $\mathbf{f}-\mathbf{i}$. Developing conidia attach to conidiogenous cells. $\mathbf{j}-\mathbf{m}$. Conidia. Scale bars: $\mathrm{a}=500 \mu \mathrm{m}, \mathrm{b}=200 \mu \mathrm{m}, \mathrm{c}=50 \mu \mathrm{m}, \mathrm{d}=20 \mu \mathrm{m}, \mathrm{e}=10 \mu \mathrm{m}, \mathrm{f}-\mathrm{m}=5 \mu \mathrm{m}$.

angularis, with a thick, hyaline inner layer with cells of textura angularis. Conidiophores reduced to conidiogenous cells. Conidiogenous cells 19-21 $\times 16-20 \mu \mathrm{m}$, cylindrical to ampulliform, holoblastic, phialidic, discrete, determinate, hyaline, smooth, formed from the inner layer of the pycnidial wall. Conidia 35-46 $\times 19-24 \mu \mathrm{m}$ (mean $=40.38 \times 22.02 \mu \mathrm{m}, \mathrm{n}=20$ ), ellipsoid to oblong, muriform, with 3 transverse and 2 longitudinal septa, continuous, straight, occasionally slightly curved, golden to dark brown, smooth-walled.

Culture characteristics - Colonies on PDA slow growing, reaching $2 \mathrm{~cm}$ diam. after 1 week at $18{ }^{\circ} \mathrm{C}$, thin mycelium, circular, rough margin white at first, white from the top, pale brown from reverse after 1 week, flat or effused on the surface. Hyphae septate branched, hyaline, thin.

Material examined - Italy, Province of Forlì-Cesena [FC], Corniolo - Santa Sofia on dead branch of Pinus nigra (Pinaceae), 7 December 2013, Erio Camporesi, IT 1564 (MFLU 15-0735, holotype) (HKAS 88743); living cultures MFLUCC 14-0457, GUCC 60. 


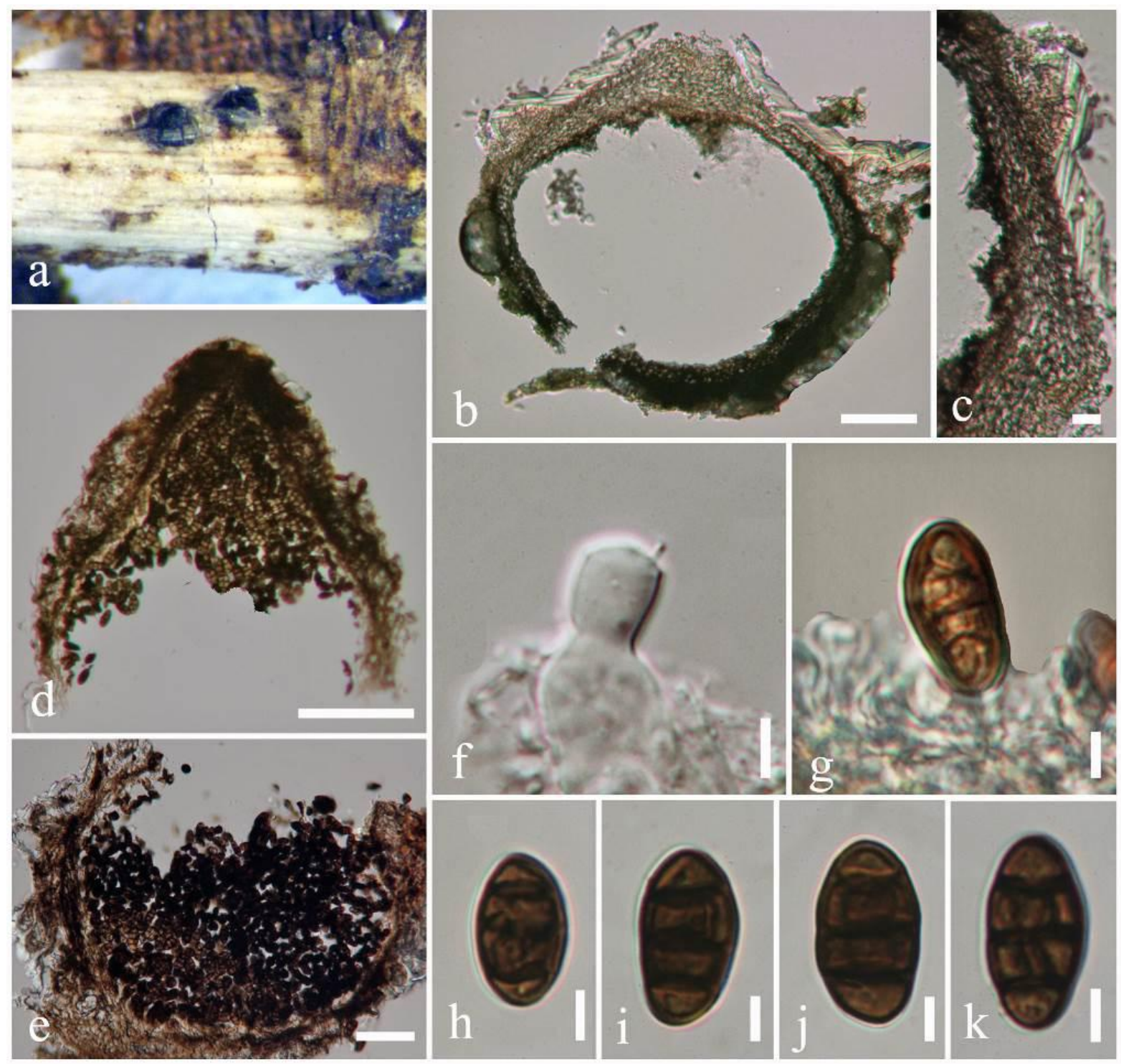

Fig. 3 - Pseudocamarosporium pinicola (holotype) a. Conidiomata on dead stem of Pinus nigra. b, d, e. Vertical sections of pycnidia. c. Conidioma wall. f-g. Conidiogenous cells and conidiogenesis. h-k. Conidia. Scale bars: $\mathrm{b}=200 \mu \mathrm{m}, \mathrm{c}=40 \mu \mathrm{m}, \mathrm{d}-\mathrm{e}=100 \mu \mathrm{m}, \mathrm{f}-\mathrm{k}=10 \mu \mathrm{m}$.

Notes - Pseudocamarosporium brabeji Marinc. et al. (14-18.5 × 7-9 $\mu \mathrm{m}$ fide Marincowitz et al. 2008). Camarosporium pini (Westend.) Sacc. (18-20 × 9-10 $\mu \mathrm{m}$ fide Barna et al. 2010) and Camarosporium propinquum (Sacc.) Sacc. have been reported previously from Pinus spp. (both species on Pinus halepensis fide Farr \& Rossman 2016). Both these species are morphologically distinct from our collection. In molecular data analyses, our new strain resides in Pseudocamarosporium sensu stricto, but is phylogenetically distinct from P. propinquum (Fig. 1). Therefore, our collection is introduced as a new species.

\section{Discussion}

Morphological plasticity in coelomycetous genera confuses the classification and has been discussed by Wijayawardene et al. (2012a). Thus genera such as Camarosporium, Coniothyrium, and Phoma have been treated as polyphyletic in Dothideomycetes (Wijayawardene et al. 2012b). The heterogenic nature of camarosporium-like taxa was discussed by Sutton (1980) who pointed out the difference in conidiogenesis in C. quaternatum (Hazsl.) Schulz, the type species of Camarosporium and C. propinquum (Sacc.). Wijayawardene et al. (2014c) confirmed Sutton's observation through DNA sequence analyses, which showed that $C$. propinquum grouped in 
Didymosphaeriaceae, Massarineae, while Camarosporium sensu stricto groups in Pleosporinae (Wijayawardene et al. 2014a). Pseudocamarosporium was therefore introduced to accommodate $C$. propinquum with four other species. Molecular analyses indicated that camarosporium-like taxa formed distinct phylogenetic lineages in Pleosporales hence Neocamarosporium, Paracamarosporium and Suttonomyces were introduced (Crous et al. 2013, Wijayawardene et al. 2014c, 2015). In this paper, we introduce two new species of Pseudocamarosporium which are distinct in both morphology and phylogeny (Fig. 1).

Crous et al. (2015) showed that Paraconiothyrium africanum clustered with Pseudocamarosporium species, thus, they transferred to Pseudocamarosporium. Hence, the Pseudocamarosporium comprises both camarosporium-like and paraconiothyrium-like species.

\section{Acknowledgements}

Nalin N. Wijayawardene thanks the Mushroom Research Foundation (MRF), Chiang Rai Province, Thailand for providing a Postgraduate Scholarship and National Science Funding of China (no. 31560489). Kevin D. Hyde thanks the Chinese Academy of Sciences, project number 2013T2S0030, for the award of Visiting Professorship for Senior International Scientists at Kunming Institute of Botany. Erio Camporesi appreciates Giancarlo Lombardi for his invaluable help in the collecting programme and identifying host plants. Yong Wang and Yan-Ling An would like to thank the project of Science and Technology Department of Guizhou Province [2012]401206. Further, we would like to thank Mae Fah Luang University grant for studying Dothideomycetes (no. 56101020032).

\section{References}

Alves A, Correia A, Luque J, Phillips A.J.L. 2004 - Botryosphaeria corticola, sp. nov. on Quercus species, with notes and description of Botryosphaeria stevensii and its anamorph, Diplodia mutila. Mycologia 96, 598-613.

Alves A, Phillips AJL, Henriques I, Correia A. 2000 - Evaluation of amplified ribosomal DNA restriction analysis (ARDRA) as a method for the identification of Botryosphaeria species. FEMS Microbiology Letters 245, 221-229.

Ariyawansa HA, Tanaka K, Thambugala KM, Phookamsak R, Tian Q, Camporesi E, Hongsanan S, Monkai J, Wanasinghe DN, Chukeatirote E, Kang JC, Xu JC, Mckenzie EHC, Jones EBG, Hyde KD. 2014 - A molecular phylogenetic reappraisal of the Didymosphaeriaceae (= Montagnulaceae). Fungal Diversity 68, 69-104.

Barna M, Sedmák R, Marušák R. 2010 - Response of European beech radial growth to shelterwood cutting. Folia Oecologica 37(2), 125-136.

Chomnunti P, Hongsanan S, Aguirre-Hudson B, Tian Q, Peršoh D, Dhami MK, Alias AS, Xu J, Liu X, Stadler M, Hyde KD. 2014 - The sooty moulds. Fungal Diversity 66, 1-36.

Crous PW, Schumacher RK, Wingfield MJ, Lombard L, Giraldo A, Christensen M, Gardiennet A, Nakashima C, Pereira O, Smith AJ, Groenewald JZ (2015) Fungal Systematics and Evolution: FUSE 1 - Sydowia 67, 81-118.

Crous PW, Wingfield MJ, Guarro J, Cheewangkoon R, Van Der Bank M, Swart WJ, Stchigel AM, Cano-Lira JF, Roux J, Madrid H, Damm U, Wood AR, Shuttleworth LA, Hodges CS, Munster M, de Jesús Yáñez-Morales M, ZúñigaEstrada L, Cruywagen EM, de Hoog GS, Silvera C, Najafzadeh J, Davison EM, Davison PJN, Barrett MD, Barrett RL,Manamgoda DS, Minnis AM, Kleczewski NM, Flory SL, Castlebury LA, Clay K, Hyde KD, MaússeSitoe SND, Shuaifei Chen, Lechat C, Hairaud M, Lesage-Meessen L, Pawłowska J, Wilk M, Śliwińska-Wyrzychowska A, Mętrak M, Wrzosek M, Pavlic-Zupanc D, Maleme HM, Slippers B, Mac Cormack WP, Archuby DI, Grünwald NJ, Tellería MT, Dueñas M, Martín MP, Marincowitz S, de Beer ZW, Perez CA, Gené J, Marin-Felix Y, Groenewald JZ. 2013 - Fungal Planet description sheets, 154-213. Persoonia 31, 188-296. 
Huelsenbeck JP, Ronquist F. 2001 - MRBAYES: Bayesian inference of phylogenetic trees. Bioinformatics 17, 754-755.

Hyde KD, Jones EBG, Liu JK, Ariyawansa H, Boehm E, Boonmee S, Braun U, Chomnunti P, Crous PW, Dai DQ, Diederich P, Dissanayake A, Doilom M, Doveri F, Hongsanan S, Jayawardena R, Lawrey JD, Li YM, Liu YX, Lücking R, Monkai J, Muggia L, Nelsen MP, Pang KL, Phookamsak R, Senanayake I, Shearer CA, Suetrong S, Tanaka K, Thambugala KM, Wijayawardene NN, Wikee S, Wu HX, Zhang Y, Aguirre-Hudson B, Alias SA, Aptroot A, Bahkali AH, Bezerra JL, Bhat DJ, Camporesi E, Chukeatirote E, Gueidan C, Hawksworth DL, Hirayama K, Hoog SD, Kang JC, Knudsen K, Li WJ, Li XH, Liu ZY, Mapook A, McKenzie EHC, Miller AN, Mortimer PE, Phillips AJL, Raja HA, Scheuer C, Schumm F, Taylor JE, Tian Q, Tibpromma S, Wanasinghe DN, Wang Y, Xu JC, Yan J, Yacharoen S, Zhang M (2013) Families of Dothideomycetes. Fungal Diversity 63, 1-313.

Index Fungorum 2016. http://www.indexfungorum.org/Names/Names.asp

Jayasiri SC, Hyde KD, Ariyawansa HA, Bhat DJ, Buyck B, Cai L, Dai YC, Abd-Elsalam KA, Ertz D, Hidayat I, Jeewon R, Jones EBG, Bahkali AH, Karunarathna SC, Liu JK, Luangsa-ard JJ, Lumbsch HT, Maharachchikumbura SSN, McKenzie EHC, Moncalvo JM, Ghobad-Nejhad M, Nilsson H, Pang KL, Pereira OL, Phillips AJL, Raspé O, Rollins AW, Romero AI, Etayo J, Selçuk F, Stephenson SL, Suetrong S, Taylor JE, Tsui CKM, Vizzini A, Abdel-Wahab MA, Wen TC, Boonmee S, Dai DQ, Daranagama DA, Dissanayake AJ, Ekanayaka AH, Fryar SC, Hongsanan S, Jayawardena RS, Li WJ, Perera RH, Phookamsak R, de Silva NI, Thambugala KM, Tian Q, Wijayawardene NN, Zhao RL, Zhao Q, Kang JC, Promputtha I. 2015 - The Faces of Fungi database: fungal names linked with morphology, phylogeny and human impacts. Fungal Diversity 74, 3-18.

Katoh K, Misawa K, Kuma K, Miyata T. 2002 - MAFFT: a novel method for rapid multiple sequence alignment based on fast Fourier transform. Nucleic Acids Research 30, 30593066.

Katoh K, Toh H, 2008 - Recent developments in the MAFFT multiple sequence alignment program. Briefings in Bioinformatics 9, 276-285.

Liu JK, Hyde KD, Gareth EBG, Ariyawansa HA, Bhat DJ, Boonmee S, Maharachchikumbura SSN, McKenzie EHC, Phookamsak R, Phukhamsakda C, Shenoy BD, Abdel-Wahab MA, Buyck B, Chen J, Chethana KWT, Singtripop C, Dai DQ, Dai YC, Daranagama DA, Dissanayake AJ, Doilom M, D'souza MJ, Fan XL, Goonasekara ID, Hirayama K, Hongsanan S, Jayasiri SC, Jayawardena RS, Karunarathna SC, Li WJ, Mapook A, Norphanphoun C, Pang KL, Perera RH, Peršoh D, Pinruan U, Senanayake IC, Somrithipol S, Suetrong S, Tanaka K, Thambugala KM, Tian Q, Tibpromma S, Udayanga D, Wijayawardene NN, Wanasinghe DN, Wisitrassameewong K, Zeng XY, Abdel-Aziz FA, Adamč́́k S, Bahkali AH, Boonyuen N, Bulgakov T, Callac P, Chomnunti P, Greiner K, Hashimoto A, Hofstetter V, Kang JC, Lewis $\mathrm{D}$, Li XH, Liu XZ, Liu ZY, Matsumura M, Mortimer PE, Rambold G, Randrianjohany E, Sato G, Sri-Indrasutdhi V, Tian CM, Verbeken A, von Brackel W, Wang Y, Wen TC, Xu JC, Yan JY, Zhao RL, Camporesi E. Fungal diversity notes 1-110: taxonomic and phylogenetic contributions to fungal species. Fungal Diversity 72, 1-197.

Marincowitz S, Crous PW, Groenewald JZ, Wingfield MJ. 2008 - Microfungi occurring on the Proteaceae in the fynbos. 1-166pp.

Nylander JAA. 2004 - MrModeltest 2.0. Program distributed by the author. Evolutionary Biology Centre, Uppsala University.

Phillips AJL, Alves A, Pennycook SR, Johnston PR, Ramaley A, Akulov A, Crous PW. 2008 Resolving the phylogenetic and taxonomic status of dark-spored teleomorph genera in the Botryosphaeriaceae. Persoonia 21, 29-55.

Rambaut A. 2012 - FigTree version 1.4.0.http://tree.bio.ed.ac.uk/software/figtree/

Rannala B, Yang Z. 1996 - Probability distribution of molecular evolutionary trees: a new method of phylogenetic inference. Journal of Molecular Evolution 43, 304-311. 
Silvestro D, Michalak I. 2010 - raxmlGUI: a graphical front-end for RAxML http://sourceforge.net/projects/raxmlgui/. Accessed August 2010.

Stamatakis A. 2006 - RAxML-VI-HPC: Maximum likelihood-based phylogenetic analyses with thousands of taxa and mixed models. Bioinformatics 22, 2688-2690.

Sutton BC. 1980 - The Coelomycetes. Fungi imperfecti with Pycnidia, Acervuli and Stromata. Commonwealth Mycological Institute, Kew, UK.

Vilgalys R, Hester M. 1990 - Rapid genetic identification and mapping of enzymatically amplified ribosomal DNA from several Cryptococcus species. Journal of Bacteriology 172, 42384246.

White TJ, Bruns T, Lee S, Taylor J. 1990 - Amplification and direct sequencing of fungal ribosomal RNA genes for phylogenetics. In: Innis, M.A. Gelfand, D.H. Sninsky, J.J. \& White, T.J. (Eds.) PCR protocols: a guide to methods and applications. Academic Press, San Diego, California, U.S.A., pp. 315-322.

Wijayawardene DNN, Mckenzie EHC, Chukeatirote E, Wang Y, Hyde KD. 2012c - Coelomycetes. Cryptogamie Mycologie 33(3), 215-244.

Wijayawardene DNN, Mckenzie EHC, Hyde KD. 2012a - Towards incorporating anamorphic fungi in a natural classification - checklist and notes for 2011. Mycosphere 3(2), 157-228.

Wijayawardene DNN, Udayanga D, Mckenzie EHC, Wang Y, Hyde KD. 2012b - The future of coelomycete studies. Cryptogamie Mycologie 33, 381-391.

Wijayawardene NN, Bhat DJ, Hyde KD, Camporesi E, Wikee S, Chethana KWT, Tangthirasunun N, Wang Y, 2014a -Camarosporium sensu stricto in Pleosporinae, Pleosporales with two new species. Phytotaxa 183(1), 16-26.

Wijayawardene NN, Crous PW, Kirk PM, Hawksworth DL, Boonmee S, Braun U, Dai DQ, D'souza MJ, Diederich P, Dissanayake A, Doilom M, Hongsanan S, Jones EBG, Groenewald JZ, Jayawardena R, Lawrey JD, Liu J-K, Luecking R, Madrid H, Manamgoda DS, Muggia L, Nelsen MP, Phookamsak R, Suetrong S, Tanaka K, Thambugala KM, Wanasinghe DN, Wikee S, Zhang Y, Aptroot A, Ariyawansa HA, Bahkali AH, Bhat DJ, Gueidan C, Chomnunti P, De Hoog GS, Knudsen K, Li W-J, McKenzie EHC, Miller AN, Phillips AJL, Piatek M, Raja HA, Shivas RS, Slippers B, Taylor JE, Tian Q, Wang Y, Woudenberg JHC, Cai L, Jaklitsch WM, Hyde KD. 2014b - Naming and outline of Dothideomycetes-2014 including proposals for the protection or suppression of generic names. Fungal Diversity 69, 1-55.

Wijayawardene NN, Hyde KD, Bhat DJ, Camporesi E, Schumacher RK, Chethana KWT, Wikee S, Bahkali AH, Wang Y. 2014c - Camarosporium-like species are polyphyletic in Pleosporales; introducing Paracamarosporium and Pseudocamarosporium gen. nov. in Montagnulaceae. Cryptogamie Mycologie 35(2), 177-198.

Wijayawardene NN, Hyde KD, Bhat DJ, Goonasekara ID, Nadeeshan D, Camporesi E, Schumacher RK, Wang Y. 2015 - Additions to brown spored coelomycetous taxa in Massarineae, Pleosporales; introducing Phragmocamarosporium gen. nov. and Suttonomyces gen. nov. Cryptogamie Mycologie 36(2), 213-224

Zhaxybayeva O, Gogarten JP. 2002 - Bootstrap, Bayesian probability and maximum likelihood mapping: exploring new tools for comparative genome analyses. BMC Genomics 3, 4. 